\title{
Finite density simulations using a determinant estimator
}

\author{
Andrei Alexandru*, Anyi Li, Keh-Fei Liu \\ Department of Physics and Astronomy, University of Kentucky, Lexington KY 40506, USA \\ E-mail: alexanepa.uky.edu, anyiliepa.uky.edu, liuepa.uky.edu
}

\begin{abstract}
Previous investigations have shown that the canonical approach to simulating QCD at finite density is promising. The algorithm we used in our earlier work employs an exact calculation of the fermionic determinant which limits the size of the lattices we can simulate. Interesting questions can only be answered if we simulate at larger volume. In this paper we explore an algorithm, Hybrid Noisy Monte Carlo, that employs a determinant estimator rather than an exact calculation. We first present the technical aspects of the estimator, check that the algorithm is correct by comparing it with our previous study, and then discuss its merits. We will also discuss the challenges faced when simulating larger lattice volumes.
\end{abstract}

The XXV International Symposium on Lattice Field Theory

July 30-4 August 2007

Regensburg, Germany

\footnotetext{
*Speaker.
} 


\section{Introduction}

Simulating QCD at non-zero baryon density remains one of the challenges of lattice QCD. Direct Monte Carlo simulations are hindered by the fact that the fermionic determinant is complex when the chemical potential is different from zero. The common solution of separating out a complex phase from the integrand and introducing it in the observable fails due to the sign problem and overlap problem. A solution to the overlap problem was proposed in [1]; the method proposed starts from the canonical partition function rather than the usual approach based on the grand canonical one. For two degenerate flavors of quarks the canonical partition function is:

$$
Z_{C}(V, T, k)=\int \mathscr{D} \mathrm{U} e^{-S_{G}(U)} \operatorname{det}_{k} M(U)^{2},
$$

where

$$
\operatorname{det}_{k} M(U)^{2} \equiv \frac{1}{2 \pi} \int_{0}^{2 \pi} \mathrm{d} \phi e^{-i k \phi} \operatorname{det} M(U, \mu=i \phi T)^{2} .
$$

With the above choice the total net quark number is fixed, i.e. $n_{u}+n_{d}=k$. Simulating the above action involves computing the determinant for every phase $\phi$; this is not feasible. We replace the continuous Fourier transform, $\operatorname{det}_{k} M^{2}$ with a discrete one, $\operatorname{det}_{k}^{\prime} M^{2}$. The error due to this approximation is discussed in [2] and further analyzed in [3]. In the canonical approach we still have to integrate over a complex integrand; the fermion contribution $\operatorname{det}_{k} M(U)^{2}$ is complex when $k \neq 0$. In our study [2] we used a Monte Carlo method to generate an ensemble based on the weight

$$
W(U) \propto e^{-S_{G}(U)}\left|\operatorname{Re}_{\operatorname{det}_{k}^{\prime} M(U)^{2}}\right|,
$$

and the removed phase is reintroduced in the observable measurement. To generate an ensemble with this weight we use Metropolis method where the accept/reject step is based on the ratio of determinants. For more details the reader is referred to the original paper [2].

Calculating the determinant of the fermionic matrix exactly is time consuming; it is only feasible for small lattices. To move to larger lattices we will use a method that involves an estimator for the determinant. This method was proposed in [1]; in this work we will investigate it numerically.

\section{The algorithm}

In this section we will present the algorithm used in our simulations. We start by rewriting the partition function

$$
\begin{aligned}
Z_{C}(V, T, k) & =\int \mathscr{D} \mathrm{U} e^{-S_{G}(U)} \operatorname{det}_{k}^{\prime} M(U)^{2} \\
& =\int \mathscr{D} \mathrm{U} \mathscr{D} \xi e^{-S_{G}(U)} \operatorname{det} M(U)^{2} f_{k}(U, \xi)
\end{aligned}
$$

where

$$
\int \mathscr{D} \xi g(U, \phi, \xi)=\frac{\operatorname{det} M\left(U_{\phi}\right)^{2}}{\operatorname{det} M(U)^{2}}
$$

and

$$
f_{k}(U, \xi)=\frac{1}{N} \sum_{\phi_{i}} e^{-i k \phi_{i}} g\left(U, \phi_{i}, \xi\right)
$$


We introduce the estimator $f_{k}(U, \xi)$ by rewriting the integral in terms of a new, auxiliary field $\xi$. This auxiliary field plays the role of a stochastic variable. The other important feature is that we separate out $\operatorname{det} M(U)^{2}$ and we estimate the ratio $\operatorname{det}_{k}^{\prime} M(U)^{2} / \operatorname{det} M(U)^{2}$. There are two reasons for this separation: it improves the acceptance rate and also the ratio estimator is more precise than the estimator for the determinant alone.

Since the determinant projection is complex its estimator will also be complex. In order to simulate it, we need to separate a positive part out and fold the remaining phase in the observables. We will then generate ensembles based on the weight:

$$
W(U, \xi) \propto e^{-S_{G}(U)} \operatorname{det} M(U)^{2}\left|f_{k}(U, \xi)\right| .
$$

The algorithm proceeds in two steps: we first update the gauge fields $U \rightarrow U^{\prime}$ using an HMC update that satisfies detailed balance with respect to $e^{-S_{G}(U)} \operatorname{det} M(U)^{2}$ and accept this change with the probability baseon on the ratio $\left|\frac{f_{k}\left(U^{\prime}, \xi\right)}{f_{k}(U, \xi)}\right|$. The reason for separating out $\operatorname{det} M(U)^{2}$ is that the new gauge field proposal takes into account the fermionic contribution. As showed in [2] this improves the acceptance rate of this step dramatically.

The second step in the updating process is to refresh the auxiliary variables $\xi$. To implement the Noisy Monte Carlo algorithm [4], another accept/reject step is used where a new proposal $\xi \rightarrow \xi^{\prime}$ is accepted with probability based on the ratio $\left|\frac{f_{k}\left(U, \xi^{\prime}\right)}{f_{k}(U, \xi)}\right|$. It is easy to check that this algorithm satisfies the detailed balance with respect to the weight $W(U, \xi)$ in Eq. (2.4).

\section{The estimator}

The most interesting technical aspect is setting up the estimator. In this section we describe how to set up $g(U, \phi, \xi)$, an unbiased estimator for the determinant ratio [5]. We start by rewriting Eq. (2.2):

$$
\int \mathscr{D} \mathrm{U} \mathscr{D} \xi g(U, \phi, \xi)=e^{2 \operatorname{Tr}\left(\ln \mathrm{M}\left(\mathrm{U}_{\phi}\right)-\ln \mathrm{M}(\mathrm{U})\right)} .
$$

The construction of the estimator occurs in steps. First approximate $\ln M$ using a Pade approximation:

$$
\ln M \approx b_{0} I+\sum_{i=1}^{K} \frac{b_{i}}{c_{i}+M}
$$

For our simulations, we used a Pade approximation of order $K=30$.

We then set up an estimator for the exponent of the right hand side of Eq. (3.1). For the trace, we use an unbiased estimator based on $Z(4)$ noise. We generate random vectors $\eta$ and use

$$
h(\eta)=2 \eta^{\dagger}\left[\ln M\left(U_{\phi}\right)-\ln M(U)\right] \eta,
$$

as an estimator for $2 \operatorname{Tr}\left(\ln \mathrm{M}_{\phi}-\ln \mathrm{M}\right)$. It is easy to show that when the elements of $\eta$ are picked with equal probability from $Z(4)=\{1,-1, i,-i\}$, the estimator $h(\eta)$ is unbiased. Unfortunately, without improvement this estimator has a large variance. In [5] it is shown that the variance is proportional with the magnitude of the off-diagonal elements of the matrix. The suggested strategy is to subtract from the matrix of interest matrices that are traceless, so that the estimator remains unbiased, and that emulate the off-diagonal structure of the original matrix, so that the variance is 
reduced. We carry out this program using the set of matrices $D^{k}$, where $D$ is the Wilson hopping matrix. The order of the improvement is given by the largest $k$ in the set. The variance is reduced exponentially with the order of improvement as can be seen from Fig. 1. The only problem is that $\operatorname{TrD}^{\mathrm{k}}$ is non-zero when $k$ is even and larger than 2; for these orders we use the traceless matrix $D^{k}-\frac{1}{N} \operatorname{Tr} \mathrm{D}^{\mathrm{k}}$, where $N$ is the dimension of the matrix. The computational burden is then to compute $\operatorname{TrD}^{\mathrm{k}}$; to evaluate this we have to compute all closed loops of $k$ steps. For $k=4$, we have 6 such loops at every point corresponding to different plaquette orientations and, on a $4^{4}$ lattice, 4 Polyakov loops wrapping around the lattice in different directions. As we increase $k$ the number of such loops increases quickly, 112 for $k=6,2884$ for $k=8$ and 84360 for $k=10$. For the purpose of testing, we carried out the calculation up to order $k=11$, but this is very expensive. In our simulations we used only improvement up to order 9.


Figure 1: Improvement of the trace estimator. Left panel: average and error of 1000 different noises with different level of improvement; the line corresponds to the exact value. Right panel: the variance as a function of the improvement order.

Once the estimator for the exponent is set up, we have to design an unbiased estimator for the exponential function. Using the exponent estimator $h$, we follow [6] and write

$g[h]\left(\eta_{1}, \eta_{2}, \ldots\right)=1+h\left(\eta_{1}\right)+H\left(\theta_{1}-\frac{1}{2}\right) h\left(\eta_{1}\right) h\left(\eta_{2}\right)+H\left(\theta_{1}-\frac{1}{2}\right) H\left(\theta_{2}-\frac{2}{3}\right) h\left(\eta_{1}\right) h\left(\eta_{2}\right) h\left(\eta_{3}\right)+\ldots$,

where $H(x)$ is the step function which is 0 for $x<0$ and equals 1 otherwise. The numbers $\theta_{i}$ are random variables uniformly distributed over the interval $[0,1]$. The series looks infinite, but as soon as one of the step functions is zero we can stop evaluating the following terms. In general this happens quite fast and the average number of exponent estimators $h\left(\eta_{i}\right)$ that needs to be evaluated is $e-1 \approx 1.72$. It is easy to show that this estimator averages to the desired value.

We have now an unbiased estimator for $\frac{\operatorname{det} M_{\phi}^{2}}{\operatorname{det} M^{2}}$. The only problem is that it has a very large variance. To understand how to address this problem, we plot in Fig. 2 the variance of the estimator $g$ as a function of the mean value and variance of the estimator $h$. You can see that the variance of the estimator grows very quickly when the mean value or the variance of $h$ are larger than 1 . Unfortunately, we cannot control how big these quantities are; they are dictated by how large the lattice is and the ensemble temperature. 

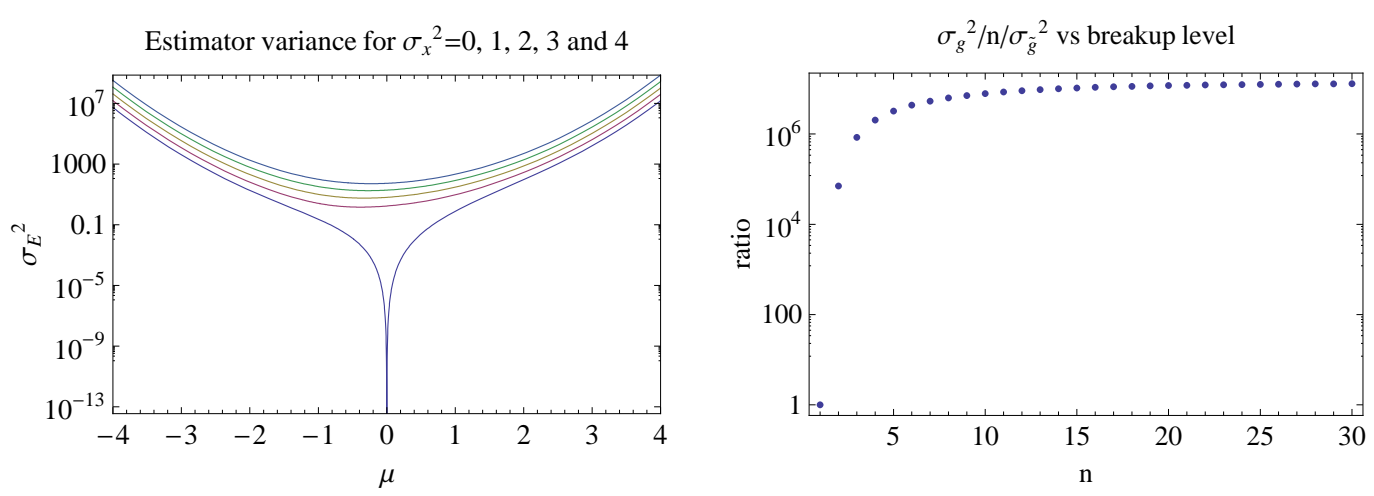

Figure 2: Exponential estimator variance. Left panel: $\mu$ denotes the average value of the $h$ estimator and $\sigma_{x}^{2}$ its variance; the variance of the $g$ estimator is denoted by $\sigma_{E}^{2}$. Right panel: we plot the increase in variance when using naive averaging of $n$ estimates of $g$ compared to the variance of $\tilde{g}$.

To address this problem, we use an estimator breakup: imagine that instead of $h(\eta)$ in Eq. (3.4) we use $h(\eta) / n$. Then the $g$ estimator would average to the $n^{\text {th }}$ root of the determinant ratio. If we take $n$ independent estimates of this new $g$ and multiply them together we get a new estimator for the ratio $\tilde{g}$. This estimator has a smaller variance than $g$, but it is $n$ times more expensive to evaluate. To confirm that this trick is useful, we compare the variance decrease with the one that we would get by just evaluating the average of $n$ independent estimates of $g$. This average costs the same as $\tilde{g}$ and its variance is $n$ times smaller than the variance of $g$. In Fig. 2 we plot this ratio (i.e. $\frac{\sigma_{g}^{2} / n}{\sigma_{\tilde{g}}^{2}}$ ) for a case when $\langle h\rangle=5$ and $\left\langle h^{2}\right\rangle=5.1$ (small variance for the exponent). We see from the figure that if we use the naive averaging, the variance would be increased by about a million times when the breakup level is greater than 5 .

The first lesson is that the breakup estimator has a much better variance than the native averaging. The second important thing to notice is that when the breakup level is greater than the average value of $h$ the payoff levels off; this means that further reductions in variance come at the same rate as statistical reduction. We derive then a rule of the thumb to help us in setting up the breakup level: the breakup level, $n$, should be set so that most of the estimates of $h$ are smaller than $n$. In practice this means that we have to setup $n$ larger than the average value of $h$ plus a few standard deviations.

\section{Algorithm check}

To verify that the algorithm is correct we run a set of simulations on $4^{4}$ lattices at the same parameters as the ensembles we generated in our previous work [2]. Before we present the results, we want to discuss the simulations. We had run simulations at $\beta=5.10,5.15,5.20,5.25$ and 5.30. These simulations correspond to temperatures between $153 \mathrm{MeV}$ and $189 \mathrm{MeV}$. We chose these simulation points since they are close to the transition temperature and this is the area that we are interesting in scanning in our future work. For each temperature, we ran three simulations for zero, one and two baryon numbers. The hopping parameter was set to $\kappa=0.158$ which corresponds to a pion mass of approximatively $1 \mathrm{GeV}$. We tuned the HMC trajectories length so that the acceptance rate of the gauge update is about $50 \%$. 
The only new parameter that we had to set up is the breakup level. As discussed in the previous section, the breakup level needs to be setup such that most of the estimates are smaller than $n$. To simplify things we took the ensembles we generated in our previous study and, for each configuration, we evaluated the determinant for all the phases we used in that study. For each configuration, we computed $\max _{\phi} \operatorname{Tr} \ln M_{\phi}-\min _{\phi} \operatorname{Tr} \ln M_{\phi}$ and we found it to be bound by 15 . To be on the safe side, we set the breakup level to $n=20$.

To compare the results of the current simulations with the ones employing an exact calculation of the determinant, we chose to measure the absolute value of the Polyakov loop and the baryon chemical potential. In Fig. 3 we compare the results of our runs with the results we got from our previous study; the results agree quite well and this leads us to conclude that the method and implementation are sound.
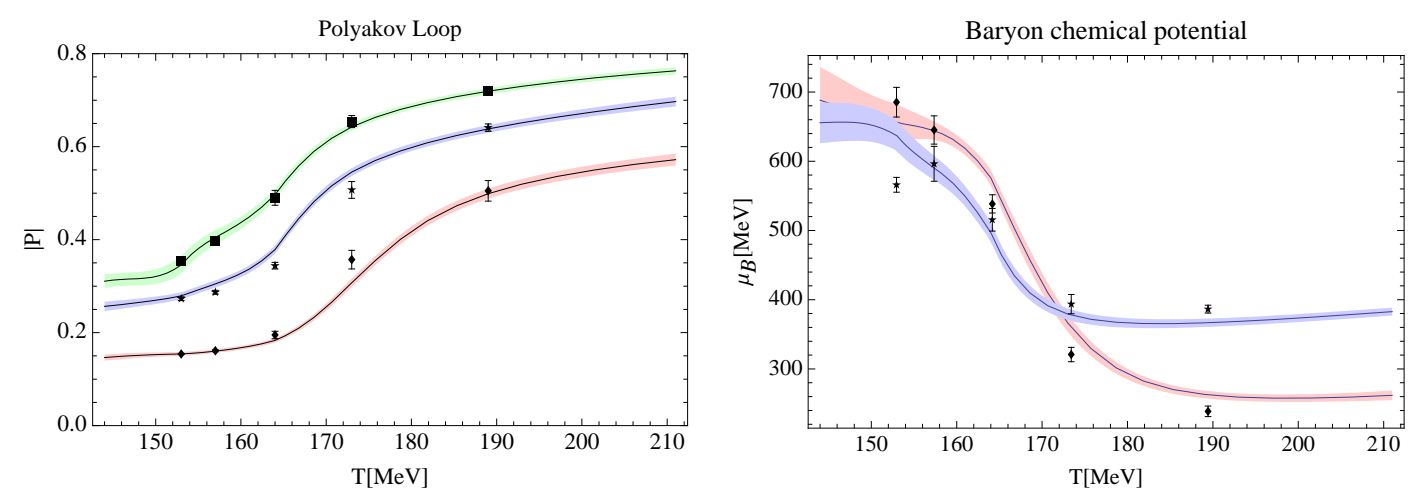

Figure 3: Checking the algorithm. Left panel: Absolute value of the Polyakov loop; the solid lines are the result of our previous work and the symbols are the result of our current simulation. From bottom up the curves correspond to 0,1 and 2 baryon simulations. Right panel: Chemical potential - the lines are our previous results and the symbols are the results of our current simulations. The red curve represents the difference in free energy between the 1 and 0 baryon simulations and the blue curve corresponds to the difference between 2 and 1.

\section{Volume dependence}

Our first simulations on $4^{4}$ lattices confirm the correctness of the algorithm. However, to produce interesting results we need to move to larger volumes. The main drive behind the idea of using an estimator in our simulation is that the exact calculations of the determinant scale quite poorly with the size of the lattice - as we increase the lattice volume $V$ the cost of the calculation increases with $V^{3}$ whereas the estimator calculation was expected to scale like $V$ (the cost of computing $\left(M+b_{i}\right)^{-1} \eta$ in Eq. (3.2) needed to approximate $\left.\ln M \eta\right)$.

It turns out that the estimator is actually more expensive due to the determinant breakup: the cost of the calculation increases linearly with the level of breakup and, as we discussed in a previous section, the level of breakup needs to be adjusted as the fluctuations of $\operatorname{Tr} \ln \mathrm{M}_{\phi}$ become larger. To determine how these fluctuations scale, we ran simulations on $4^{4}, 6 \times 4^{3}, 6^{2} \times 4^{2}$ and $6^{3} \times 4$ lattices. In Fig. 4 we show how the average and the variance of the fluctuations of the exponent $h$ vary with volume. It is easy to see that they increase linearly with the volume. We conclude then that the cost of computing the estimator scales with $V^{2}$. 



Figure 4: Volume dependence of the fluctuations. Left panel: the mean value of the fluctuations as a function of volume. Right panel: the variance of the fluctuations as a function of volume.

\section{Conclusions}

This work is part of a larger effort aimed at simulating QCD at non-zero baryon density. We chose to investigate an approach based on the canonical partition function mainly because this method should not have an overlap problem. Previous investigations $[2,7]$ have shown it to be very promising. In this paper, we analyze a method based on an estimator [1]. The main technical issue we discuss is the implementation of the estimator: we describe the details of our implementation and show that it is correct by running simulations on $4^{4}$ lattices. The results of these simulations compare well with the ones we obtained using a different method [2].

We also look at the performance of the estimator and how it scales with the volume. We find that the estimator is more expensive that originally expected. However, even on $4^{4}$ lattice the algorithm performs better than the exact method: 100 seconds for computing the estimator twice (once for the gauge update and once for the stochastic field update) versus 140 seconds for the exact calculation. Furthermore, when moving to larger lattices the gains will increase since the estimator calculation scales as $V^{2}$ compared to $V^{3}$ for the exact calculation.

Our next goal is to run simulations on $6^{3} \times 4$ lattices and look for a phase transition signal at non-zero baryon density. This was shown to be feasible [7] and we plan to perform a similar analysis for our simulations. In this paper we show that the best method to attack larger volume simulations is the estimator method and we hope to report soon on simulations at larger volumes.

\section{References}

[1] K. F. Liu, QCD and Numerical Analysis Vol. III, Springer, New York, 2005, p. 101.

[2] A. Alexandru, M. Faber, I. Horváth, K. Liu, Phys. Rev. D72, (2005) 114513 [hep-lat / 0507020 ].

[3] Anyi Li, Andrei Alexandru, Keh-Fei Liu, these proceedings.

[4] L.Lin, K.F. Liu, and J. Sloan, Phy. Rev., D61, (2000) 074505.

[5] C. Thron, S.J. Dong, K. F. Liu, H. P. Ying Phys. Rev. D57 (1998) 1642 [hep-lat/ 9707001$].$

[6] G. Bhanot, A. D. Kennedy, Phys. Lett. 157B, (1985) 70.

[7] Ph. de Forcrand, S.Kratochvila, Nucl. Phys. B (Proc. Suppl.) 153 (2006) 62 [hep-lat / 0602024 ]. 